\title{
LncRNA NEAT1 promotes endometrial cancer cell proliferation, migration and invasion by regulating the miR-144-3p/EZH2 axis
}

\author{
Wei Wang ${ }^{1}$, Liang Ge², Xiao-Juan Xu¹, Ting Yang ${ }^{1}$, Yue Yuan¹, Xiao-Ling Ma1 ${ }^{1}$, Xue-Hong Zhang ${ }^{1}$

\begin{abstract}
${ }^{1}$ The Reproductive Medicine Special Hospital of the $1^{\text {st }}$ Hospital of Lanzhou University, Key Laboratory for Reproductive Medicine and Embryo, Lanzhou, Gansu Province, P. R. China

2 Department of Anesthesiology, Gansu Province Maternity and Child-care Hospital, Lanzhou, Gansu Province, P. R. China
\end{abstract}

Radiol Oncol 2019; 53(4): 434-442.

Received 22 May 2019

Accepted 21 September 2019

Correspondence to: Dr. Xue-Hong Zhang \& Dr. Wei Wang, The Reproductive Medicine Special Hospital of the $1^{\text {st }}$ Hospital of Lanzhou University, Key Laboratory for Reproductive Medicine and Embryo, No.1, Donggangxi Road, Chengguan District, Lanzhou 730000, Gansu Province, P. R. China. E-mail: zhangxueh@lzu.edu.cn and wangwei83819@163.com

Disclosure: No potential conflicts of interest were disclosed.

Background. Endometrial cancer (EC) is one of the most common gynaecological tumours in the worldwide. Long non-coding RNA (IncRNA) nuclear enriched abundant transcript 1 (NEAT1) promotes cell proliferation, migration and invasion in EC cells. However, the molecular mechanisms of NEATI in EC have not been fully clarified. We conducted this study to reveal the function of NEATI in EC tissues and cell lines.

Materials and methods. Cancer and adjacent tissues were collected from EC patients. HEC-1A and Ishikawa cells were cultured in vitro. NEAT1 expression was downregulated by transfecting small hairpin RNA (shRNA) and miR-144$3 p$ was overexpressed by transfecting miR-144-3p mimics. Cell proliferation was detected by MTT assay and colony formation assay. Cell migration and invasion abilities were assessed by transwell assay. A dual-luciferase reporter assay was used to verify the relationship among NEAT1, EZH2, and miR-144-3p. The expression level of EZH2 was measured by Western blot and qPCR.

Results. NEATI was highly expressed in EC tissues and cells. Knockdown of NEAT1 inhibited the proliferation, migration and invasion of EC cells. Additionally, NEAT1 acted as a ceRNA of miR-144-3p, leading to EZH2 upregulation. Overexpression of miR-144-3p suppressed the proliferation and invasion of EC cells.

Conclusions. NEATI promotes EC cells proliferation and invasion by regulating the miR-144-3p/EZH2 axis.

Key words: endometrial cancer; NEAT1; miR-144-3p; EZH2

\section{Introduction}

Endometrial cancer (EC), one of the most common gynaecological tumours originating in the uterus, has increasing incidence rates, as shown by American Cancer Society statistics. ${ }^{1}$ The relative 5-year survival relative rate for $\mathrm{EC}$ is only $77 \%$, and approximately 11,350 women died of uterine cancer in 2016 in the U.S. ${ }^{2}$ Consequently, determining the molecular mechanisms underlying the development of EC is of great importance.

Long non-coding RNAs (lncRNAs) are defined as the RNA transcripts, that are longer than 200 nucleotides but do not encode proteins. LncRNAs play unique regulatory roles in a variety of biological pathways, such as cardiovascular, reproductive, inflammatory, and metabolic functions and DNA repair processes. ${ }^{3-5}$ Emerging evidence has confirmed the carcinogenic or anticancer effects of IncRNAs in cancer development. ${ }^{6,7}$ LncRNA nuclear enriched abundant transcript 1 (NEAT1) is dysregulated in multiple types of malignancies, including bladder cancer, lung cancer, breast cancer and colorectal cancer. ${ }^{8}$ It has been reported that overexpression of NEAT1 promotes cell proliferation, migration and invasion in EC and associates 
with clinical progression. ${ }^{9}$ In addition, NEAT1 can regulate the $\mathrm{Wnt} / \beta$-catenin signalling pathway by targeting miR-214-3p or miR-146b-5p in EC. ${ }^{10,11}$ The results mentioned above reveal the important roles of NEAT1 in EC progress. However, its mechanism in EC remains largely unknown.

The histone methyltransferase EZH2 has been identified as a clinically relevant biomarker associated with cancer. Dysregulation of EZH2 was found in various cancers. For example, EZH2 can promote tumour growth by increasing angiogenesis in pituitary adenoma. ${ }^{12} \mathrm{EZH} 2$ expressed is also dysregulated in EC and is related to high grade of EC. ${ }^{13}$ It was demonstrated that EZH2 is the target of miR-144-3p in osteosarcoma cells and negatively correlates with the expression of miR-144-3p. ${ }^{14}$ Whether miR-144-3p is involved in the progression of EC through EZH2 remains to be explored. In our previous work, we predicted that NEAT1 may target miR-144-3p. Thus, we speculated that NEAT1 might regulate EC cells proliferation, migration and invasion via the miR-144-3p/EZH2 axis.

In this study, we found that NEAT1 could bind to $\mathrm{miR}-144-3 \mathrm{p}$ to suppress its function and subsequently upregulate the expression of $\mathrm{EZH} 2$, leading to enhanced EC cells proliferation, migration, and invasion. Taken together, our findings reveal a mechanism in which the NEAT1-miR-144-3pEZH2 axis regulates proliferation, migration and invasion in EC cells, suggesting that NEAT1 may serve as a potential target for EC treatment.

\section{Materials and methods}

\section{Cell culture}

The human endometrial cancer cell lines, HEC-1-A, HEC-1B, Ishikawa (type I), RL-95-2, and JEC and human normal endometrial stromal cells (hESCs) were maintained in DMEM (Gibco, USA) with $10 \%$ foetal bovine serum (FBS, Gibco, USA). The cells were cultured at $37^{\circ} \mathrm{C}$ with $5 \% \mathrm{CO}_{2}$.

\section{Cancer and adjacent tissue collection}

All the EC and adjacent normal endometrial tissues were collected from EC patients at the First Hospital of Lanzhou University. The EC tissues $(\mathrm{n}=36)$ and tumour adjacent tissues $(\mathrm{n}=36)$ were collected during surgery and then rapidly frozen with liquid nitrogen and stored at $-80^{\circ} \mathrm{C}$. This study was approved by the Ethics Committee of the Reproductive Medicine Special Hospital of the First Hospital of Lanzhou University.

\section{Quantitative real-time PCR (qRT-PCR)}

Total RNA extraction was performed using Trizol reagent (Invitrogen USA), and a TaqMan Reverse Transcription Kit (Thermo Fisher, USA) was used for reverse transcription into cDNA according to the manufacturer's instructions. Real-time quantitative polymerase chain reaction (qPCR) was used to quantify the expression level of NEAT1 in cultured cells using an Applied Biosystems 7500 Fast Dx Real-Time PCR instrument (Thermo Fisher, USA). SYBR Green reagent was used for qPCR in this study. GAPDH was used as an internal reference to normalize NEAT1 and EZH2 values. U6 was used as an internal reference to normalize miR-144-3p values. The following primers were used for analysis:

NEAT1, 5'-TTGGGACAGTFFACGTGTGG-3' (forward), and 5'-TCAAGTCCAGCAGAGCA-3' (reverse); miR-144-3p,

\section{5'-GGCCGGCGTACAGTATAGATGA-3'}

(forward), and 5'- GTGCAGGGTCCGAGGT-3'

(reverse);

EZH2, 5'-AAGCACAGTGCAACACCAAG-3'

(forward), and $5^{\prime}-$

CAGATGGTGCCAGCAATAGA-3' (reverse); GAPDH, 5'-TGACGTGCCGCCTGGAGAAC-3' (forward), and 5'-CCGGCATCGAAGGTGGAAGAG-3' (reverse); U6, 5'-CTCGCTTCGGCAGCACA-3' (forward), and 5' - AACGCTTCACGAATTTGCGT3'(reverse). Relative RNA levels were calculated using the $2^{-\Delta \Delta} \mathrm{Ct}$ method.

\section{Cell transfection}

Small hairpin RNA (shRNA) for NEAT1 or its respective negative control were designed, synthesized and cloned into the shRNA vector U6/GFP/ Neo plasmid (GenePharma, Shanghai, China). A miR-144-3p mimic and its control were designed and synthesized by Sangon (Shanghai, China). The validated and purified reconstructed vectors were transfected into the indicated cells by Lipofectamine 3000 (Thermo Fisher, USA) according to the manufacturer's guidelines. Briefly, 0.2 $\mu \mathrm{g}$ indicated plasmid and $0.4 \mu \mathrm{L}$ Lipofectamine 3000 were mixed with $5 \mu \mathrm{L}$ Opti-MEM medium (Thermo Fisher, USA), and then, Opti-MEM medium containing plasmid was added to the other Opti-MEM medium containing Lipofectamine 3000. Subsequently, this mixture was added to cells whose density was approximately $70 \%$. 


\section{Dual-luciferase reporter assay}

To verify the binding ability of NEAT1 to miR-144$3 p$ and miR-144-3p to EZH2, we performed a dualluciferase reporter assay using a Promega dual-luciferase reporter assay kit (Madison, USA). Briefly, we constructed a pmiR-RB-REPORT ${ }^{\mathrm{TM}}$ (Ribobio) plasmid containing the exact sites for wild type NEAT1 and EZH2 3'-UTRs and the corresponding mutated sequences. The cells seeded in 96-well plates were co-transfected with the indicated plasmids and miR-144-3p mimic or NC duplex (Gene Pharma). After $48 \mathrm{~h}$, the cells were harvested, and luciferase activity was measured using a dual-luciferase reporter assay kit (Promega Corporation) and a multi-plate reader (Synergy 2, Bio Tek).

\section{MTT assay}

An MTT assay was performed to measure cell proliferation. Cells were cultured in 96-well plates (1 $\times 10^{4}$ per well). MTT ( $0.5 \mathrm{mg} / \mathrm{mL}$, Gibco, USA) was added to the medium. Then, the plate was incubated at $37^{\circ} \mathrm{C}$ for $4 \mathrm{~h}$ and the supernatant was discarded. Next, $150 \mu$ L DMSO (Thermo Fisher, USA) was added to each well and the OD490 nm value was measured immediately using a microplate reader (3100, Thermo Fisher, USA).

\section{Colony formation assay}

A colony formation assay was used to determine cell proliferation. HEC-1-A and Ishikawa cells
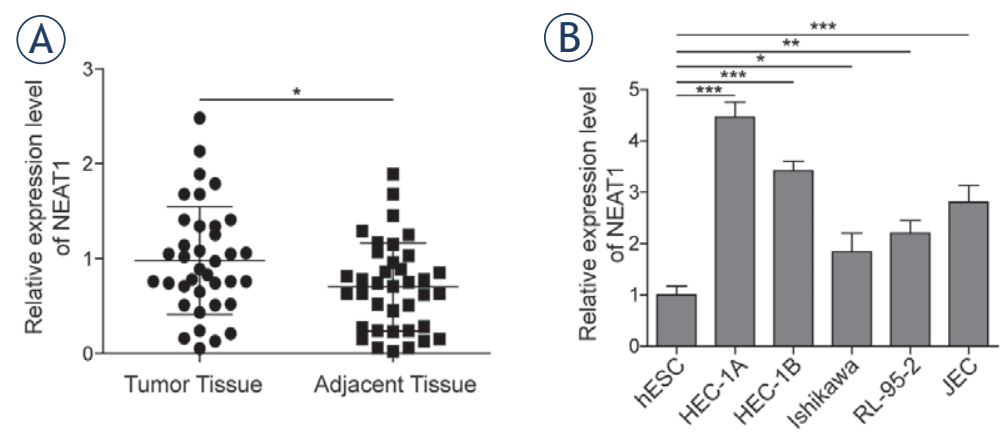

FIGURE 1. NEATI was highly expressed in EC tissues and cells. (A) NEATI was highly expressed in EC tissues. The transcription level of the NEATI gene in EC tissues was measured by GRT-PCR. Cancer tissues and adjacent tissues were from human EC patients. (B) The transcription of NEATI was upregulated in various EC cell lines. QRTPCR was used to examine the expression of NEATI in various EC cell lines (HEC-1A, HEC-1B, Ishikawa, RL-95-2, and JEC), and hESCs, which were used as a control group.

${ }^{*}$ indicates $P<0.05 ; * *$ indicates $P<0.01 ; * *$ indicates $P<0.001$. The Data are presented as the mean \pm SD. in logarithmic growth were digested by trypsin (Gibco, USA) and then resuspended in DMEM containing 10\% FBS. Cells were seeded in culture dishes at a density of 500 cells per dish. Then, the cells were cultured at $37^{\circ} \mathrm{C}$ and $5 \% \mathrm{CO}_{2}$ until visible colonies were formed. The culture medium was discarded, and the cells were fixed using methanol and stained with a crystal violet solution (Gibco, USA). The number of colonies was counted.

\section{Transwell assay}

The migration and invasion assays were performed using transwells. For this assay, 24-well plates with $8-\mu \mathrm{m}$ pore polycarbonate membranes (BD Biosciences, USA) were used. The upper side of the membranes was coated with Matrigel $(20 \mu \mathrm{g} /$ well, BD Biosciences, USA) and then air-dried for $1 \mathrm{~h}$ at $37^{\circ} \mathrm{C}$. EC cells $\left(2 \times 10^{5}\right)$ in $200 \mu \mathrm{L}$ of FBS-free medium were placed in the upper chamber, which was uncoated (migration assay) or coated (invasion assay). The lower chamber was filled with medium with $10 \%$ FBS as a chemoattractant. After 48 $h$ of incubation, the cells on the upper surface of the membrane were removed by gentle scrubbing with a cotton swab. The membranes were fixed in a stationary liquid of $95 \%$ ethanol and $5 \%$ acetic acid for $30 \mathrm{~min}$ and stained with a crystal violet solution. The number of cells on the lower surface of the membrane in 5 random visual fields (magnification, $\times 200$ ) was then counted using an Eclipse TE2000-U inverted microscope.

\section{Western blot analysis}

A protein detection kit (Thermo Fisher, USA) was used to measure the amount of protein. Equivalent amounts of protein samples were separated on 8-10\% SDS-PAGE and transferred to polyvinylidene difluoride membranes (PVDF, Thermo Fisher, USA). After blocking with $0.1 \%$ Tween 20 and $5 \%$ skimmed milk, the protein-containing PVDF membranes were incubated for $12 \mathrm{~h}$ in solutions containing primary antibodies obtained from Abcam (anti-EZH2, ab186006; anti-GAPDH, ab9485) at $4^{\circ} \mathrm{C}$. Then, secondary antibodies were incubated for $2 \mathrm{~h}$. A western blotting kit (Thermo Fisher Scientific, USA) was used to detect the signals.

\section{Statistical analysis}

Statistical analysis was performed using GraphPad Prism. The continuous variables are shown as the 
(A)

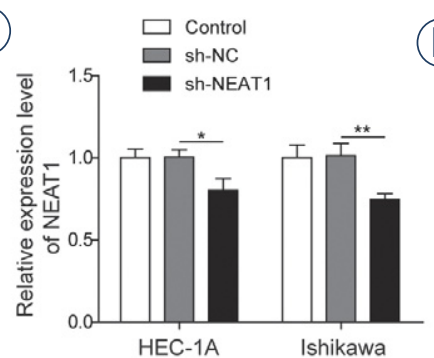

(B)

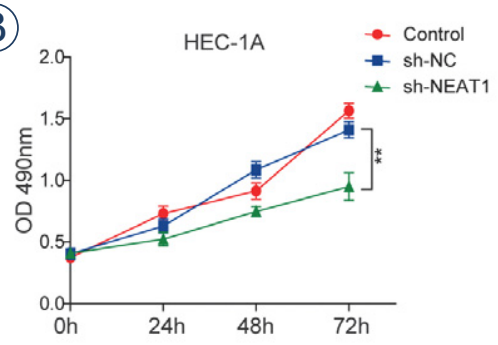

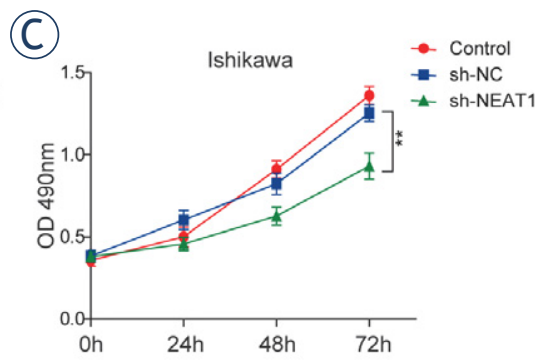

(D)

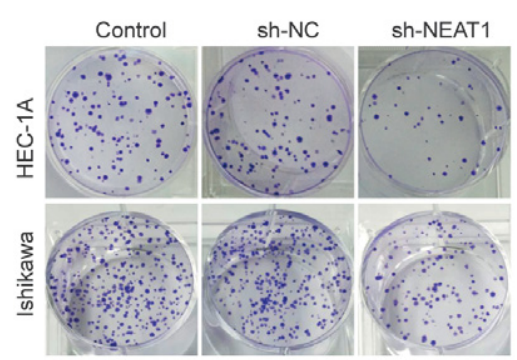

(F)

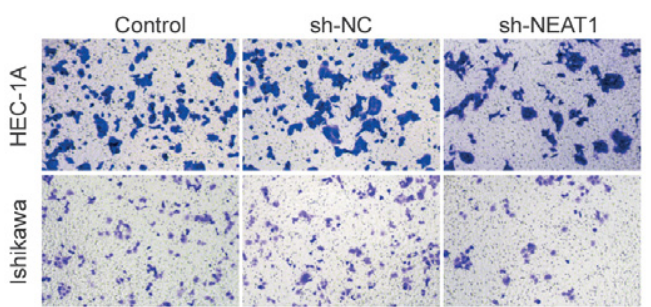

(H)

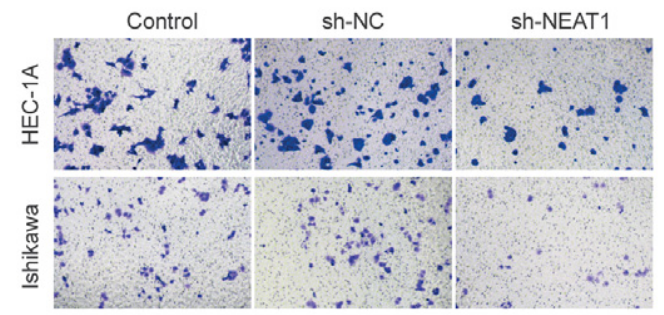

(E)

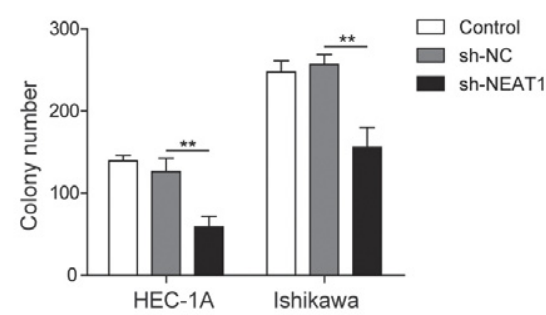

(G)

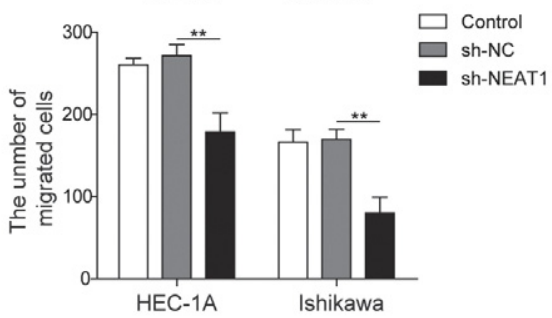

(1)

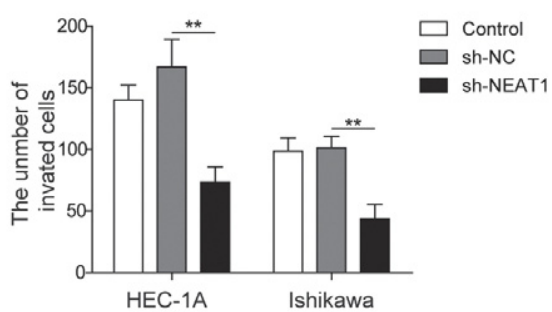

FIGURE 2. NEATI promoted EC cell proliferation, migration, and invasion. (A) Effects of NEATI-shRNA on NEATI expression. The transcription of NEATI was measured by QRT-PCR after sh-NEATI and sh-NC were transfected into HEC-1A and Ishikawa cells for $48 \mathrm{~h}$ respectively. $(B, C)$ Effects of NEAT1 knockdown on the proliferation of EC cells (HEC-1A and Ishikawa). The MTT assay results showed the effects on cell growth as measured by cell vitality on three consecutive days. (D, E) Effects of NEATl knockdown on the cell proliferation of EC cells. A colony formation assay was used to detect cell proliferation. Figure 2D is one representative result of the colony formation assay and Figure $2 \mathrm{E}$ shows one quantitative result repeated at least three times. $(\mathrm{F}, \mathrm{G}) \mathrm{Effects}$ of NEATI knockdown on the migration of EC cells. A transwell assay was used to measure the cell migration ability. Figure $2 \mathrm{~F}$ is a representative result from the transwell assays, and Figure $2 \mathrm{G}$ shows one quantitative result with at least three replicates. ( $\mathrm{H}, \mathrm{I})$ Effects of NEATI knockdown on the invasion of EC cells. A transwell assay was used to measure the cell invasion ability. Figure $2 \mathrm{H}$ is a representative result from the transwell assays and Figure 21 shows one quantitative result with at least three replicates.

* indicates $P<0.05$; ** indicates $P<0.01$. The data are presented as the mean \pm SD.

mean \pm standard deviation (SD), and their differences were analysed by Student's $t$ test or ANOVA. $P$ values less than 0.05 predicted the statistical significance of the results. Each assay was performed in triplicate.

\section{Results}

\section{NEAT1 is highly expressed in EC tissues and cells}

We first evaluated the expression of NEAT1 in cancer tissues and adjacent tissues derived from EC 
(A)

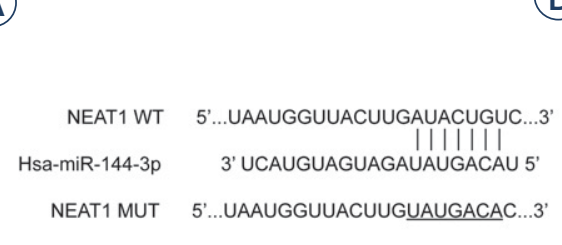

(D)

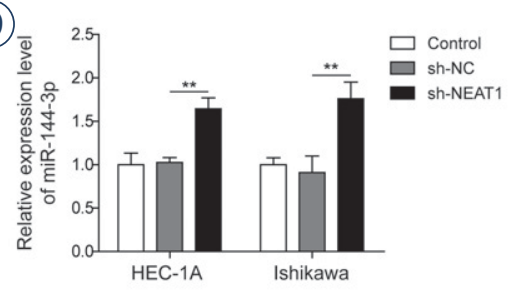

(B)

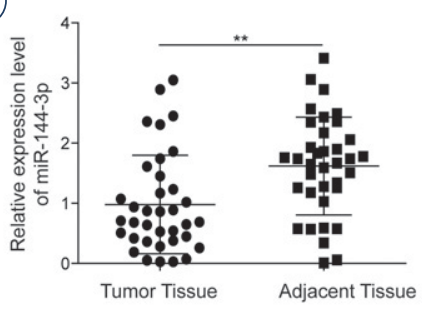

(E)

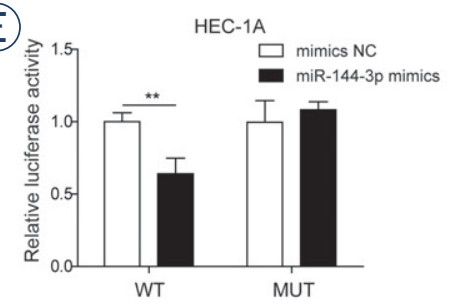

(C)
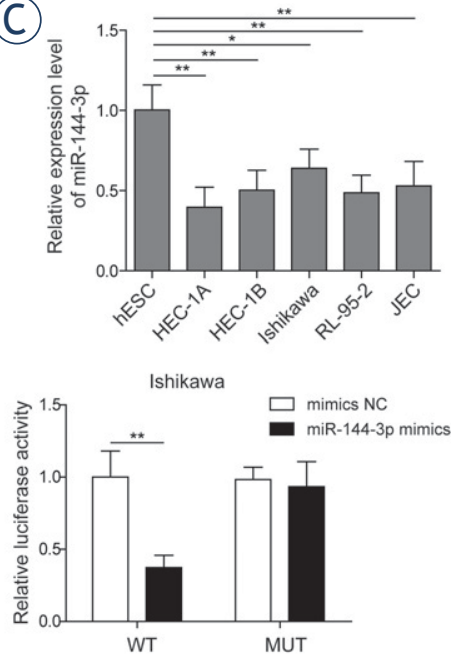

FIGURE 3. NEATI is a molecular sponge of miR-144-3p. (A) Sequences of miR-144-3p and wild-type NEATl (NEATI WT) and its mutant (NEATI MUT). NEATI WT can target miR-144-3p, whereas NEAT1 MUT lost this ability. (B) miR-144-3p was downregulated in EC tissues. The transcription level of miR-144-3p in EC tissues was measured by qRT-PCR. Cancer tissues and adjacent tissues were from human EC patients. (C) The transcription of miR-144-3p was upregulated in various EC cell lines. qRT-PCR was used to examine the expression of miR-144-3p in various EC cell lines (HEC-1A, HEC-1B, Ishikawa, RL-95-2 and JEC), and hESCs, which were used as a control group. (D) Effects of NEATl knockdown on miR-144-3p expression. The transcription of miR-144-3p was measured by qRTPCR after sh-NEATl and sh-NC were transfected into HEC-1A and Ishikawa cells for 48 h. (E) NEATl targeted miR-144-3p. A dual luciferase activity assay was used to evaluate the binding ability of NEATI WT and NEATI MUT to miR-144-3p.

* indicates $P<0.05$; ** indicates $P<0.01$. The data are presented as the mean \pm SD.

patients by qRT-PCR. As shown in Figure 1A, we found that the expression of NEAT1 was prominently higher in EC tissues than in adjacent tissues $(P<0.05$, indicated a significant difference). To determine whether NEAT1 is highly expressed in various EC-related cell lines, we performed qRTPCR to detect its expression in hESCs and endometrial cancer cell lines. Interestingly, we found that the transcription levels of NEAT1 were significantly higher in EC cell lines, including HEC1A, HEC-1B, Ishikawa, RL-95-2, and JEC, than in hESCs (Figure 1B). Among these cell lines, HEC$1 \mathrm{~A}$ and Ishikawa cells had the highest and lowest expression of NEAT1, respectively, and they were selected for the following experiments.

\section{NEAT1 promotes EC cell proliferation, migration, and invasion}

To investigate whether endogenous NEAT1 plays a role in EC, we constructed a NEAT1-shRNA plasmid that efficiently downregulated NEAT1 expression after transfection into HEC-1A and Ishikawa cells (Figure 2A). MTT and colony formation assays were performed to measure the EC cell proliferation ability. According to the MTT assay, knockdown of NEAT1 significantly inhibited cell growth compared with the NC and control conditions in
HEC-1A (Figure 2 B) and Ishikawa cells (Figure 2 C). Furthermore, colony formation assays indicated that the suppression of NEAT1 reduced colony formation in HEC-1A and Ishikawa cells (Figure 2 D, E). Taken together, the MTT assay and colony formation assays results indicated that knockdown of NEAT1 inhibited EC cell proliferation.

To determine whether endogenous NEAT1 affects EC cell migration and invasion, we performed a transwell assay to detect cell migration and invasion abilities. We found that knockdown of NEAT1 attenuated the migration of HEC-1A and Ishikawa cells (Figure 2 F, G). Consistently, we also found that knockdown of NEAT1 inhibited the invasion of HEC-1A and Ishikawa cells (Figure $2 \mathrm{H}, \mathrm{I}$ ). Taken together, these data suggest that NEAT1 promotes EC cell proliferation, migration, and invasion.

\section{NEAT1 is a molecular sponge of miR-144-3p}

LncRNAs may act as sponges for direct binding to miRNA. In our previous work, we predicted that NEAT1 may target miR-144-3p using Starbase (http://starbase.sysu.edu.cn/) for bioinformatics prediction (Figure $3 \mathrm{~A}$ ). The miR-144-3p level was much lower in EC tissues than in adjacent tissues according to qRT-PCR. MiR-144-3p was also ex- 
(A)

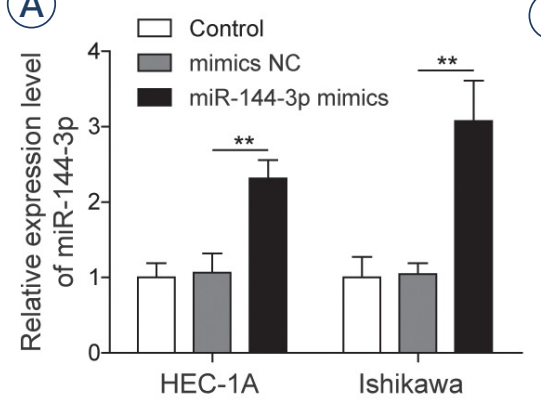

(D)

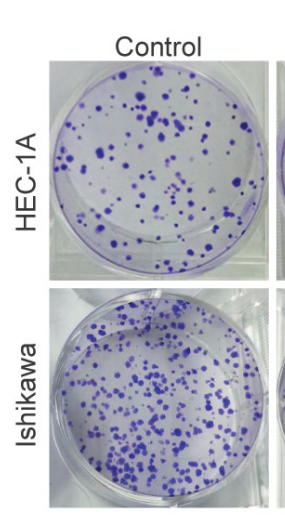

(F)

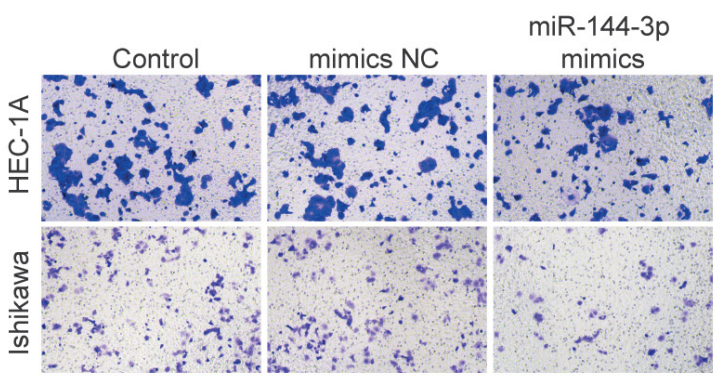

(H)

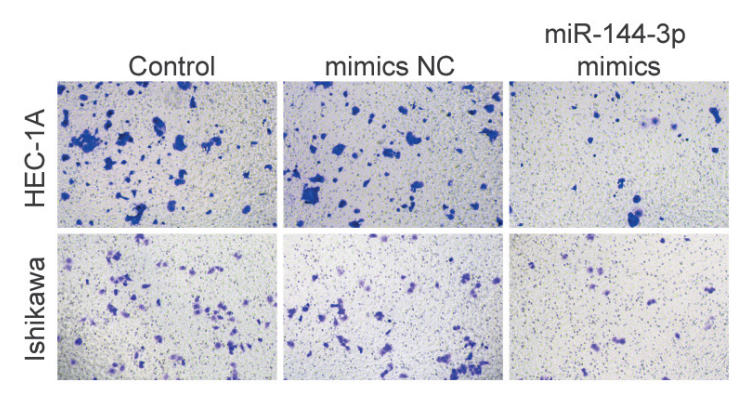

(B)

miR-144-3p

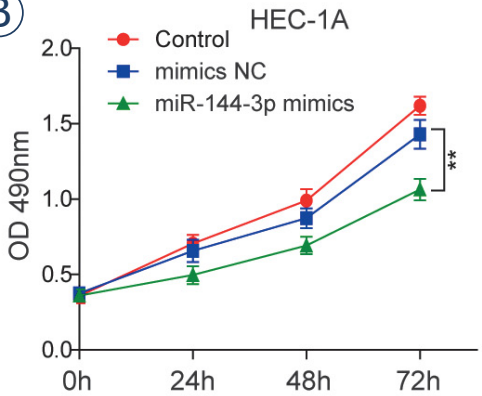

(C)

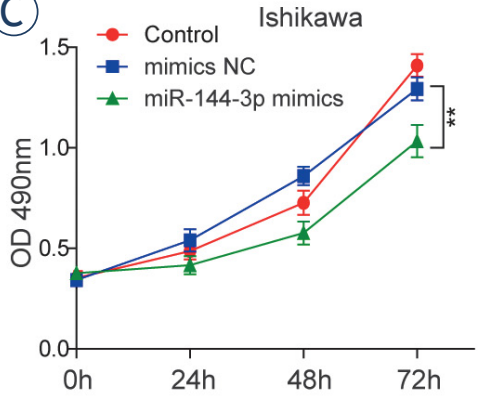

(E)

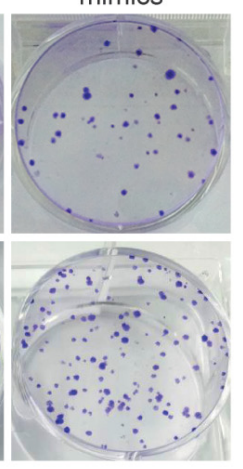

(G)
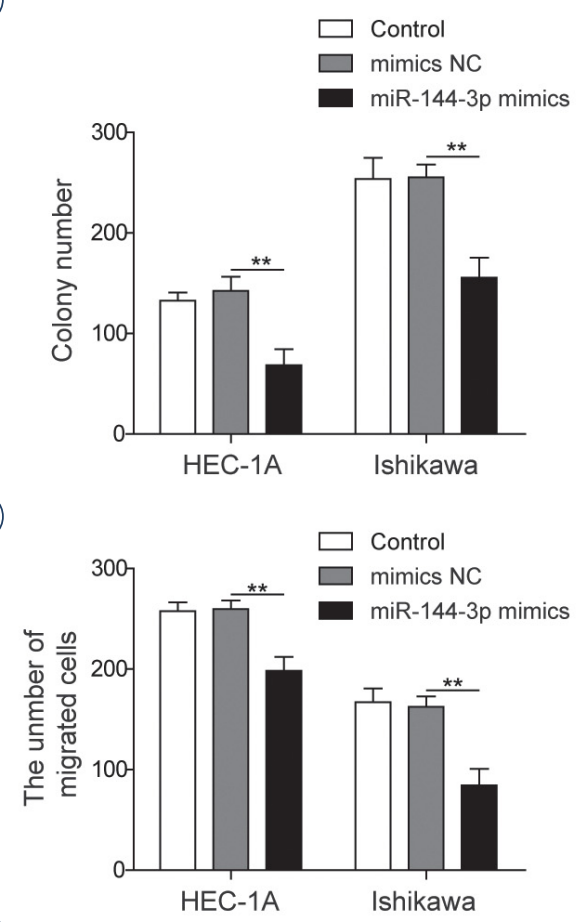

(1)

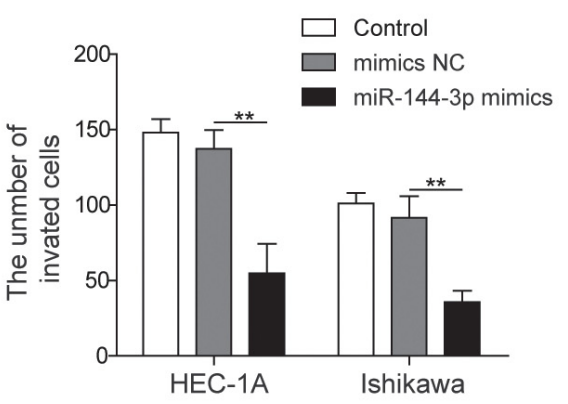

FIGURE 4. miR-144-3p inhibited EC cell proliferation, migration, and invasion. (A) Effects of the miR-144-3p mimic on miR-144-3p expression. The transcription of miR-144-3p was measured by qRT-PCR after miR-144-3p mimics or mimic NC transfection into HEC$1 \mathrm{~A}$ and Ishikawa cells for $24 \mathrm{~h}$. (B, C) Effects of the miR-144-3p mimic on the proliferation of EC cells. MTT assay results showed effects on cell growth as measured by cell vitality on three consecutive days. (D, E) Effects of the miR-144-3p mimic on the proliferation of EC cells. A colony formation assay was used to detect cell proliferation. Figure $4 D$ is a representative result from the colony formation assay and Figure 4 E shows one quantitative result repeated at least three times. (F, G) Effects of the miR-144-3p mimic on the migration of EC cells. A transwell assay was used to measure the cell migration ability. Figure $4 \mathrm{~F}$ is a representative result from the transwell assays and Figure $4 G$ shows one quantitative result with at least three replicates. $(\mathrm{H}, \mathrm{I})$ Effects of the miR-144-3p mimic on the invasion of EC cells. A transwell assay was used to measure the cell invasion ability. Figure $4 \mathrm{H}$ is a representative result from the transwell assays, and Figure 4 l shows one quantitative result with at least three replicates.

** indicates $P<0.01$. The data are presented as the mean \pm SD. 


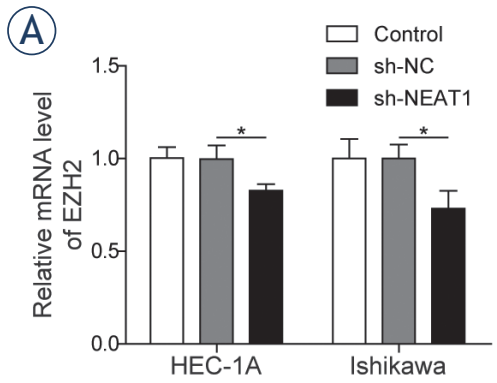

(D)

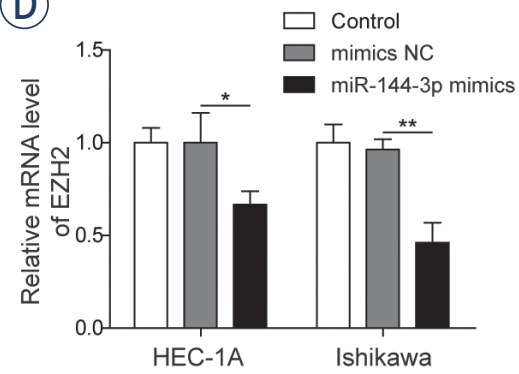

(G)
(B)

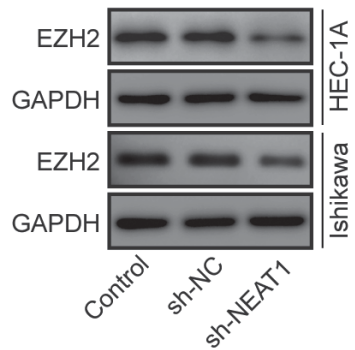

(E)

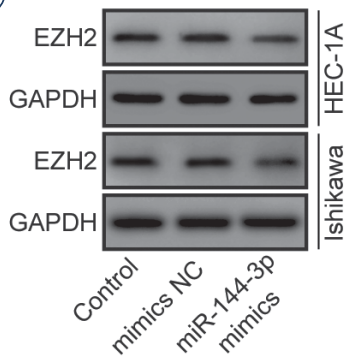

(C)

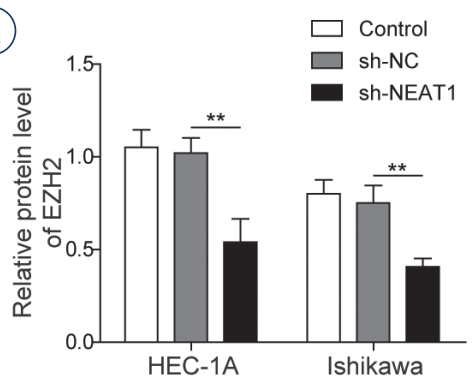

(F)
EZH2 3'UTR WT
5'... UCUGAAUUUGCAAAGUACUGUAA... $3^{\prime}$
Hsa-miR-144-3p
EZH2 3'UTR MUT
3' UCAUGUaguagaUAUGACAU 5'
5'... UCUGAAUUUGCAAAGUCGCAGUA....'
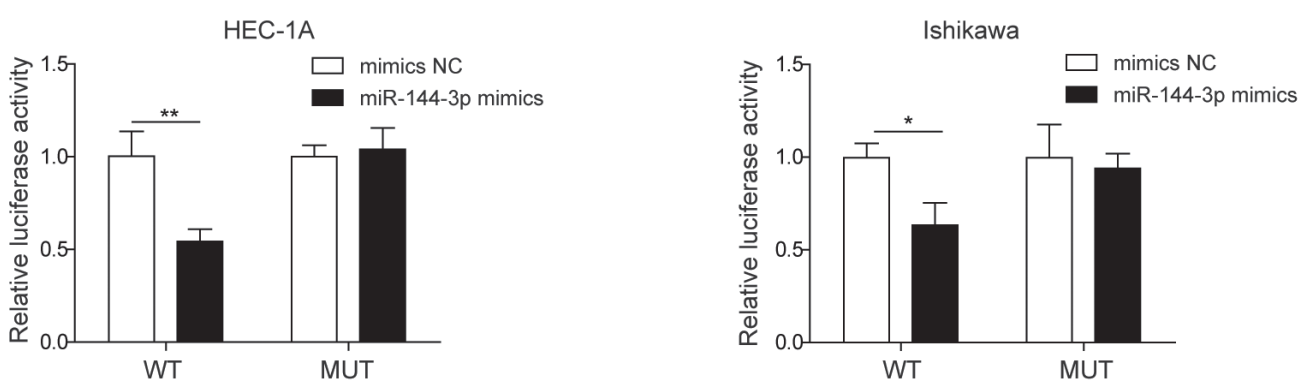

FIGURE 5. NEAT1 promoted the expression of EZH2 by miR-144-3p. (A) Effects of NEAT1 knockdown on the transcription of EZH2 in EC cells. The transcription of the EZH2 gene was measured by qRT-PCR after sh-NEATl and sh-NC were transfected into HEC-1A and Ishikawa cells for 48 h. (B, C) Effects of NEAT1 knockdown on the EZH2 protein level of in EC cells. The protein level of EZH2 was measured by Western blot after sh-NEATl and sh-NC transfection into HEC-1A and Ishikawa cells for $48 \mathrm{~h}$. The indicated antibodies were used for Western blot analysis. Quantitative Western blot results are shown in Figure 5B with GAPDH as a control. Figure 4C shows one representative result and the right part shows one quantitative result repeated at least three times. (D) Effects of miR144-3p mimic on the transcription of EZH2 in EC cells. The transcription of the EZH2 gene was measured by qPCR after miR-144-3p mimic and mimic NC transfection into HEC-1A and Ishikawa cells for $48 \mathrm{~h}$. (E) Effects of the miR-144-3p mimic on the EZH2 protein level in EC cells. The EZH2 protein level was measured by Western blot after miR-144-3p mimics or mimic NC transfection into HEC-1 A and Ishikawa cells for $48 \mathrm{~h}$. The indicated antibodies were used for Western blot analysis. (F) Sequences of miR-144-3p and wildtype EZH2 (EZH2 3'-UTR WT) and its mutant (EZH2 3'-UTR MUT). EZH2 3'-UTR WT can be targeted by miR-144-3p, whereas miR-144-3p cannot bind to EZH2 3'-UTR MUT. (G) miR-144-3p directly targeted the 3'-UTR region of EZH2. A dual luciferase activity assay was used to evaluate the binding ability of miR-144-3p to EZH2 3'-UTR WT and EZH2 3'-UTR MUT.

$*$ indicates $P<0.05, * *$ indicates $P<0.01$. The data are presented as the mean \pm SD.

pressed at lower level in EC cells than in hESCs (Figure 3 B, C). Next, we performed qRT-PCR to detect whether NEAT1 targets miR-144-3p to promote EC progression. As shown in Figure 3D, we found that knockdown of NEAT1 increased the level of miR-144-3p. To determine whether NEAT1 binds directly to miR-144-3p, we performed a dual luciferase activity assay to evaluate the binding ability by designing wild-type NEAT1
(WT-NEAT1) and a mutant (MUT-NEAT1). The luciferase signal will decrease when the lncRNA binds to its target miRNA. We found that the luciferase activity was decreased in the WT-NEAT1 and miR-144-3p mimic co-transfected group, while no significant difference was observed between the MUT-NEAT1 group and he mimic NC group (Figure 3E). These data suggested that NEAT1 could bind to miR-144-3p. 
miR-144-3p inhibits EC cell proliferation, migration, and invasion

We designed experiments to reveal the function of miR-144-3p in EC cells. First, miR-144-3p was overexpressed in EC cell lines by miR-144-3p mimic transfection (Figure 4 A). Next, EC cell proliferation was examined via MTT and colony formation methods. In HEC-1A and Ishikawa cells, the MTT assay showed that cell proliferation was significantly inhibited in the miR-144-3p mimic group compared with that in the control and NC groups (Figure $4 \mathrm{~B}, \mathrm{C}$ ). Furthermore, colony formation assays showed that the miR-144-3p mimic markedly attenuated the cell proliferation of HEC$1 \mathrm{~A}$ and Ishikawa cells compared with the control and NC groups (Figure 4 D, E). Finally, to determine whether miR-144-3p affects EC cell invasion and migration, we performed transwell assays to measure the migration and invasion abilities of EC cells. We found that the miR-144-3p mimic inhibited the migration of HEC-1A and Ishikawa cells (Figure $4 \mathrm{~F}, \mathrm{G}$ ). Consistently, we also found that the miR-144-3p mimic attenuated invasion of HEC-1A and Ishikawa cells (Figure $4 \mathrm{H}, \mathrm{I}$ ). Taken together, these data indicated that miR-144-3p inhibited EC cell proliferation, migration, and invasion.

\section{NEAT1 promotes the expression of EZH2 via miR-144-3p}

We found that knockdown of NEAT1 suppressed the transcription of the EZH2 gene in HEC-1A and Ishikawa cells (Figure $5 \mathrm{~A}$ ). Consistently, Western blot analysis showed that knockdown of NEAT1 decreased the expression of EZH2 in HEC-1A and Ishikawa cells (Figure 5 B, C). However, whether miR-144-3p regulates EZH2 in EC cells was unknown. To verify the relationship between miR144-3p and EZH2 in EC cells, we overexpressed miR-144-3p in HEC-1A and Ishikawa cells by miR-144-3p mimic transfection and evaluated the expression of EZH2 by qRT-PCR and Western blotting. The qRT-PCR results showed that the transcription of the EZH2 gene was significantly lower in the miR-144-3p mimic group than in the control and NC groups in HEC-1A and Ishikawa cells (Figure 5 D). Consistently, Western blot analysis showed that the miR-144-3p mimic decreased the expression of EZH2 in HEC-1A and Ishikawa cells (Figure 5 E). To verify whether miR-144-3p decreases EZH2 expression by binding to its 3'UTR site, we performed a dual luciferase activity assay to evaluate the binding ability and designed wild-type EZH2 (WT-EZH2) and its mutant (MUTEZH2) sequences (Figure $5 \mathrm{~F}$ ). The dual-luciferase activity assay results showed that the luciferase activity was lower in the miR-144-3p mimic group than in the NC WT-EZH2 group, while there was no difference in the MUT-EZH2 groups (Figure 5 $\mathrm{G})$. Taken together, these data suggested that miR144-3p could directly target the $3^{\prime}$-UTR region of EZH2. These data suggested that NEAT1 may act as a ceRNA of miR-144-3p, leading to increased the expression of EZH2 which is a target gene of miR144-3p.

\section{Discussion}

It is well known that lncRNAs play critical roles in various biological functions and disease processes. In cancer, lncRNAs could regulate gene expression and mediate cell signalling pathways such as p53, NF- $\kappa B, P I 3 K / A K T$ and Notch signalling. ${ }^{15}$

NEAT1 is a novel lncRNA that is localized in nuclear paraspeckles. The gene that transcribes NEAT1 is located on the familial tumour syndrome multiple endocrine neoplasia type 1 locus. ${ }^{16}$ In recent studies, high expression of NEAT1 has been found in various types of cancers, and it has been shown to serve as an oncogene. For examples, Chakravarty et al. found that NEAT1 is the most markedly overexpressed IncRNA in prostate cancer and that its expression is related to prostate cancer progression. ${ }^{17}$ Dong et al. reported that the expression levels of NEAT1 are significantly increased in early-stage EC tissue samples, and high expression levels of NEAT1 predict a poor prognosis. ${ }^{18}$ In this study, we found that NEAT1 was highly expressed in EC tissues and cell lines and that downregulation of NEAT1 in EC cells inhibited cancer cell proliferation, migration and invasion, which are consistent with of the results of Dong et al. These results suggested that NEAT1 may play carcinogenic roles in the progression of EC.

Acting as a competitive endogenous RNAs, lncRNAs regulate protein expression by binding to miRNAs. For example, NEAT1 inhibits glioma cell migration and invasion via modulating SOX2 by targeting miR-132. ${ }^{19}$ In acute lymphoblastic leukaemia, NEAT1 can regulate miR-335-3p expression, and the dysregulation of miR-335-3p is associated with poor prognosis. ${ }^{20}$ In colorectal cancer, NEAT1 could promote the tumourigenesis by sponging miR-193a-3p. ${ }^{21}$ NEAT1 regulates the Wnt/ $\beta$-catenin signalling pathway via the miR-214-3p-HMGA1 axis, leading to the promotion of cell growth, mi- 
gration and invasion in EC cells. ${ }^{11}$ In this study, we first revealed that NEAT1 directly targets miR-144$3 p$ and that knockdown of NEAT1 promotes the expression of miR-144-3p. However, the function of miR-144-3p is still unknown. Through functional experiments, we found that overexpression of miR-144-3p can inhibit EC cell proliferation, migration and invasion. These results suggest that NEAT1 may act as an oncogene by targeting miR144-3p in EC cells.

EZH2 participates in histone methylation, which regulates transcriptional repression. ${ }^{22}$ Emerging evidence has shown that EZH2 is associated with EC cell proliferation. Eskander et al. found that inhibition of EZH2 expression in EC cells significantly attenuates cell proliferation, migration, and invasion by increasing the expression of the Wnt pathway inhibitors SFRP1 and DKK3. ${ }^{23}$ Wang et al. reported that knockdown of EZH2 inhibits the proliferation of EC cells in vitro and induces apoptosis in EC cells via upregulating the expression of caspase $3 / 9$ and downregulating the expression of $\mathrm{Bcl}-$ $2 .^{24}$ Ihira et al. reported that knockdown of EZH2 suppresses EC progression via the miR-361/Twist axis. ${ }^{25}$ In this study, we first confirmed that NEAT1 could upregulate the expression of EZH2 and promote the proliferation, migration and invasion of EC cells by targeting miR-144-3p, which further confirmed the function of EZH2 in the progression of EC.

In conclusion, we demonstrated that NEAT1, as an oncogene, regulates miR-144-3p-EZH2 axis, which promotes the proliferation, migration and invasion of EC cells, providing a promising therapeutic target for EC.

\section{References}

1. Siegel RL, Miller KD, Jemal A. Cancer statistics, 2017. CA Cancer J Clin 2017; 67: 7-30. doi: 10.3322/caac.21387

2. Miller $K D$, Siegel RL, Lin $C C$, Mariotto $A B$, Kramer JL, Rowland JH, et al. Cancer treatment and survivorship statistics, 2016. CA Cancer J Clin 2016; 66: 271-89. doi: 10.3322/caac.21349

3. Li J, Meng H, Bai Y, Wang K. Regulation of IncRNA and its role in cancer metastasis. Oncol Res 2016; 23: 205-17. doi: 10.3727/096504016X1454966 7334007

4. Quinn JJ, Chang HY. Unique features of long non-coding RNA biogenesis and function. Nat Rev Genet 2016; 17: 47-62. doi: 10.1038/nrg.2015.10

5. Sun W, Yang Y, Xu C, Guo J. Regulatory mechanisms of long noncoding RNAs on gene expression in cancers. Cancer Genet 2017; 216-217: 105-10. doi: 10.1016/j.cancergen.2017.06.003

6. Jing N, Huang T, Guo H, Yang J, Li M, Chen Z, et al. LncRNA CASC15 promotes colon cancer cell proliferation and metastasis by regulating the miR4310/ LGR5/Wnt/betacatenin signaling pathway. Mol Med Rep 2018; 18: 2269-76. doi: 10.3892/mmr.2018.9191
7. Li S, Zhou J, Wang Z, Wang P, Gao X, Wang Y. Long noncoding RNA GAS5 suppresses triple negative breast cancer progression through inhibition of proliferation and invasion by competitively binding miR-196a-5p. Biomed Pharmacother 2018; 104: 451-7. doi: 10.1016/j.biopha.2018.05.056

8. Ghafouri-Fard S, Taheri M. Nuclear enriched abundant transcript 1 (NEAT1): a long non-coding RNA with diverse functions in tumorigenesis. Biomed Pharmacother 2019; 111: 51-9. doi: 10.1016/j.biopha.2018.12.070

9. Li Z, Wei D, Yang C, Sun H, Lu T, Kuang D. Overexpression of long noncoding RNA, NEAT1 promotes cell proliferation, invasion and migration in endometrial endometrioid adenocarcinoma. Biomed Pharmacother 2016; 84: 244-51. doi: 10.1016/j.biopha.2016.09.008

10. Huang $X$, Zhong $R$, He $X$, Deng $Q$, Peng $X$, Li J, et al. Investigations on the mechanism of progesterone in inhibiting endometrial cancer cell cycle and viability via regulation of long noncoding RNA NEAT1/microRNA-146b-5p mediated Wnt/beta-catenin signaling. IUBMB Life 2019; 71: 223-34. doi: 10.1002/iub.1959

11. Wang J, Zhao X, Guo Z, Ma X, Song Y, Guo Y. Regulation of NEAT1/miR-214$3 p$ on the growth, migration and invasion of endometrial carcinoma cells. Arch Gynecol Obstet 2017; 295: 1469-75. doi: 10.1007/s00404-017-4365-1

12. Liu B, Pang B, Wang $Q$, Yang $S$, Gao T, Ding $Q$, et al. EZH2 upregulation correlates with tumor invasiveness, proliferation, and angiogenesis in human pituitary adenomas. Hum Pathol 2017; 66: 101-7. doi: 10.1016/j.humpath.2017.03.028

13. Zhou J, Roh JW, Bandyopadhyay S, Chen Z, Munkarah AR, Hussein Y, et al. Overexpression of enhancer of zeste homolog $2(\mathrm{EZH} 2)$ and focal adhesion kinase (FAK) in high grade endometrial carcinoma. Gynecol Oncol 2013; 128: 344-8. doi: 10.1016/j.ygyno.2012.07.128

14. Cao J, Han X, Qi X, Jin X, Li X. TUG1 promotes osteosarcoma tumorigenesis by upregulating EZH2 expression via miR-144-3p. Int J Oncol 2017; 51: 1115 23. doi: $10.3892 /$ ijo.2017.4110

15. Peng WX, Koirala P, Mo YY. LncRNA-mediated regulation of cell signaling in cancer. Oncogene 2017; 36: 5661-7. doi: 10.1038/onc.2017.184

16. Zhang $\mathrm{Q}$, Chen $\mathrm{CY}$, Yedavalli VS, Jeang KT. NEAT1 long noncoding RNA and paraspeckle bodies modulate HIV-1 posttranscriptional expression. MBio 2013; 4: e00596-00512. e00596-00512. doi: 10.1128/mBio.00596-12

17. Chakravarty D, Sboner A, Nair SS, Giannopoulou E, Li R, Hennig S, et al. The oestrogen receptor alpha-regulated IncRNA NEAT1 is a critical modulator of prostate cancer. Nat Commun 2014; 5: 5383. doi: 10.1038/ncomms6383

18. Dong $\mathrm{P}$, Xiong $\mathrm{Y}$, Yue J, Xu D, Ihira $\mathrm{K}$, Konno $\mathrm{Y}$, et al. Long noncoding RNA NEAT1 drives aggressive endometrial cancer progression via miR-361-regulated networks involving STAT3 and tumor microenvironment-related genes. J Exp Clin Cancer Res 2019; 38: 295. doi: 10.1186/s13046-019-1306-9

19. Zhou K, Zhang C, Yao H, Zhang X, Zhou Y, Che Y, et al. Knockdown of long non-coding RNA NEAT1 inhibits glioma cell migration and invasion via modulation of SOX2 targeted by miR-132. Mol Cancer 2018; 17: 105. doi: $10.1186 / \mathrm{s} 12943-018-0849-2$

20. Pouyanrad S, Rahgozar S,\& Ghodousi ES. Dysregulation of miR-335-3p, targeted by NEAT1 and MALAT1 long non-coding RNAs, is associated with poor prognosis in childhood acute lymphoblastic leukemia. Gene 2019; 692: 35-43. doi: 10.1016/j.gene.2019.01.003

21. Yu HM, Wang C, Yuan Z, Chen GL, Ye T, Yang BW. LncRNA NEAT1 promotes the tumorigenesis of colorectal cancer by sponging miR-193a-3p. Cell Prolif 2019; 52: e12526. doi:10.1111/cpr.12526

22. Vire E, Brenner C, Deplus R, Blanchon L, Fraga M, Didelot C, et al. The polycomb group protein EZH2 directly controls DNA methylation. Nature 2006; 439: 871-4. doi: 10.1038 /nature04431

23. Eskander RN, Ji T, Huynh B, Wardeh R, Randall LM, Hoang B. Inhibition of enhancer of zeste homolog $2(\mathrm{EZH} 2)$ expression is associated with decreased tumor cell proliferation, migration, and invasion in endometrial cancer cell lines. Int J Gynecol Cancer 2013; 23: 997-1005. doi: 10.1097/ IGC.0b013e318296a265

24. Wang J, Ai Z, Chen J, Teng Y, Zhu J. Enhancer of zeste homolog 2 blockade by RNA interference is implicated with inhibited proliferation, invasion and promoted apoptosis in endometrial carcinoma. Oncol Lett 2018; 15: 9429 35. doi: $10.3892 / o l .2018 .8518$

25. Ihira K, Dong P, Xiong $\mathrm{Y}$, Watari H, Konno $\mathrm{Y}$, Hanley SJ, et al. EZH2 inhibition suppresses endometrial cancer progression via miR-361/Twist axis. Oncotarget 2017; 8: 13509-20. doi: 10.18632/oncotarget.14586 